Moroccan J. of Pure and Appl. Anal. (MJPAA)

Volume 5(1), 2019, Pages 46-62

ISSN: Online 2351-8227 - Print 2605-6364

DOI 10.2478/mjpaa-2019-0005

\title{
A Discrete Duality Finite Volume Method For Coupling Darcy and Stokes Equations
}

\author{
M. RHOUdAF ${ }^{1}$ AND N. STAÏLI ${ }^{1, a}$
}

\begin{abstract}
Авstract. We present a general finite volume method to solve a coupled Stokes-Darcy problem, we propose two domains corresponding to fluid region and porous region with a physical intersection. At the contact interface between the fluid region and the porous media we impose two conditions; the first one is the normal continuity of the velocity and the second one is the continuity of the pressure. Furthermore, due to the lack of information about both the velocity and the pressure on the interface, we will use Schwarz domain decomposition. In Darcy equations, the tensor of permeability will be considered as variable, since it depends on both the properties of the porous medium and the viscosity of the fluid. Numerical examples are presented to demonstrate the efficiency of the proposed method.
\end{abstract}

Key words and phrases. Stokes, Darcy, Coupled Problem, Finite Volume Method, Schwarz, Domain Decomposition Method

\section{Introduction}

We focus our study on the flow of a fluid in a steady state, this flow is governed by Darcy's law, coupled with Stokes equation. This model describes macroscopic properties of filtration processes that find many important applications in porous media problems such as water flowing across semi-permeable soil, transport of contaminants through rivers into the aquifers or oil filtering through sand and rocks.

Let $\Omega_{1}$ and $\Omega_{2}$ be two non-intersecting open bounded subsets of $\mathbb{R}^{2}$, with lipschitzian boundaries $\partial \Omega_{1}$ and $\partial \Omega_{2}$. We assume that their boundaries have a non-empty intersection denoted by $\Gamma:=\partial \Omega_{1} \cap \partial \Omega_{2}$ and to which we refer as the interface between $\Omega_{1}$ and $\Omega_{2}$. The remaining parts of the boundaries are $\Gamma_{1}:=\partial \Omega_{1} \mid \Gamma$ and $\Gamma_{2}:=\partial \Omega_{2} \mid \Gamma$. (see Figure 1 )

The Stokes equation govern the fluid flow in $\Omega_{1}$ :

Received May 08, 2019 - Accepted August 19, 2019.

(C) The Author(s) 2017. This article is published with open access by Sidi Mohamed Ben Abdallah University.

${ }^{1}$ Faculté des Sciences, Université Moulay Ismail, Maroc.

a e-mail:n.staili@edu.umi.ac.ma . 


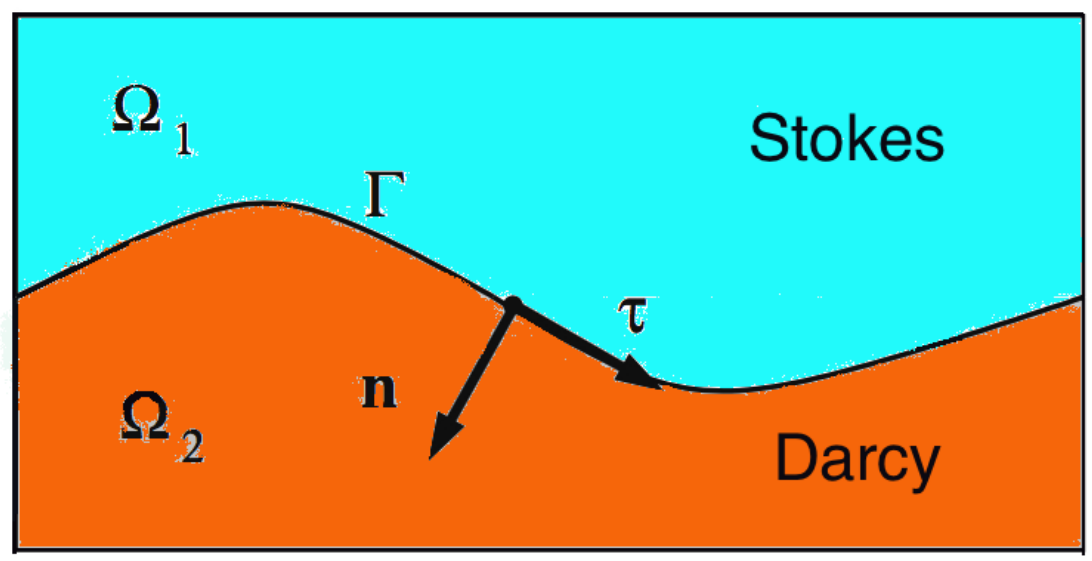

FIGURE 1. Stokes-Darcy domain

$$
\begin{array}{r}
-\nabla \cdot T\left(u_{1}, p_{1}\right)=f_{1} \text { in } \Omega_{1} \\
\nabla \cdot u_{1}=0 \text { in } \Omega_{1},
\end{array}
$$

where $u_{1}$ and $p_{1}$ are the fluid velocity and pressure, $T\left(u_{1}, p_{1}\right):=-p_{1} . I+2 \mu D\left(u_{1}\right)$ is the stress tensor, $D\left(u_{1}\right):=$ $\frac{1}{2}\left(\nabla u_{1}+\nabla u_{2}^{T}\right)$ is the deformation rate tensor and $\mu \geq 0$ is the kinematic viscosity of the fluid.

The Darcy equations govern the porous media flow in $\Omega_{2}$

$$
\begin{array}{r}
\mu u_{2}+K \nabla p_{2}=0 \text { in } \Omega_{2} \\
\nabla \cdot u_{2}=f_{2} \text { in } \Omega_{2}
\end{array}
$$

where $u_{2}$ and $p_{2}$ are the velocity and pressure, $K$ is the Darcy permeability.

On the interface $\Gamma$, we impose the following interface conditions:

$$
\begin{gathered}
u_{1} . n_{1}+u_{2} \cdot n_{2}=0 \quad \text { on } \Gamma \text {, } \\
p_{1}=p_{2} \text { on } \Gamma,
\end{gathered}
$$

where $n_{1}$ and $n_{2}$ denote the unit outward normal vector on $\partial \Omega_{1}$ and $\partial \Omega_{2}$, in particular, $n_{1}=-n_{2}$ on $\Gamma$. We assume Dirichlet boundary conditions are satisfied on $\Gamma_{1}$ and $\Gamma_{2}$ :

$$
\begin{aligned}
& u_{1}=g_{1} \quad \text { on } \Gamma_{1}, \quad u_{2}=g_{2} \quad \text { on } \Gamma_{2} \text {, } \\
& p_{1}=h_{1} \quad \text { on } \Gamma_{1}, \quad p_{2}=h_{2} \quad \text { on } \Gamma_{2} \text {. }
\end{aligned}
$$

Other type of boundary conditions could have been chosen as well.

Considerable efforts has been done to develop modelling and simulating this interaction, coupling between ground fluid and surface fluid is a very interesting subject that we found in many serious problems currently facing the world, and great deal of works has been directed to develop methods of solving Stokes-Darcy coupled problem, been motivated by a variety of applications such as bioengineering[16], industry[14, 17], environment research[10], and specially to increasing more efficient numerical methods. 
This problem has been studied from mathematical and numerical analysis viewpoint $[22,2,17,19,10,9,7]$. In[10], M. Discacciati and A. Quarteroni introduced a differential system based on the coupling of the NavierStokes equations and the Darcy equation. They formulate the problem as an interface problem, they propose a way of solving the coupled problem iteratively, by solving one problem at each step.

In[9] M. Discacciati considers a new approach to characterize preconditionners for the nonlinear Stokes-Darcy problem, then proposes a general nonlinear domain decomposition strategy for the resolution of the interface problem of nonlinear Navier-Stokes/Darcy coupling.

In[6] Prince Chidyagwai and Batrice Rivire analyzed two models for the coupled problem Stokes-Darcy, using continious finite elements in the incompressible flow region and discontinious finite elements in the porous medium.

Other works have presented the same problem with more or less generality $[21,7,4,8]$, interested in the choice of the mesh, the different conditions of the edge or the different coupling conditions on the interface.

The above mentioned works used finite element method to treat the problem (2). Our first objective is to elaborate a finite volumes scheme for the coupled Stokes-anisotropic Darcy problem, since the permeability is presented as variable in our case, the classical finite volumes method won't be able to treat this type of equation, to do so, we used the DDFV scheme. Also note that the transmission conditions between the two domains naturally intervene in the variational formulation in all works which used the finite elements method [12], what is not the case in the finite volumes method. Then our second objective is to use the Schwartz's method to overcome this difficulty.

The paper is organized as follows. In section 2, we first give the finite-volume notation associated with the particular geometry of the domain, we introduce the Finite Volume scheme and define the associated discrete operators. In section 3 we study a finite volume approximation of the incompressible Darcy-Stokes coupled problem in the whole domain $\Omega=\Omega_{1} \cup \Omega_{2}$, and we propose the method of Schwarz to treat the interface, and we give some numerical tests.

Finally, we give a conclusion and a perspective of the next problem we can hold by using an experimental condition on the interface.

\section{Framework}

2.1. Meshes and notations. In order to define a DDFV scheme, we recall here the main definitions taken from $[11,3,1]$. It is constituted by a primal mesh $\overline{\mathfrak{M}}$ and a dual mesh $\overline{\mathfrak{M}^{\star}}$.

The interior primal mesh $\mathfrak{M}$ is a set of disjoint open polygonal control volumes $\mathcal{K} \subset \Omega$ such that $\cup \overline{\mathcal{K}}=\bar{\Omega}$. We denote by $\partial \mathfrak{M}$ the set of edges of the control volumes in $\mathfrak{M}$ included in $\partial \Omega$, which we consider as degenerate control volumes.

To each control volume $\mathcal{K} \in \mathfrak{M}$, we associate a point $x_{k}$. Knowing that many choices are possible, we assume in this paper that $x_{k}$ is the mass center of $\mathcal{K}$. And to each degenerate control volume $\mathcal{K} \in \partial \mathfrak{M}$, we associate the point $x_{\mathcal{K}}$ equal the midpoint of the control volume $\mathcal{K}$.

This family of points is denoted by $\mathfrak{X}=\left\{x_{\mathcal{K}}, \mathcal{K} \in \mathcal{M} \cup \partial \mathcal{M}\right\}$.

In order to define dual control volumes, let $\mathfrak{X}^{*}$ denote the set of the vertices of the primal control volume in $\mathfrak{M}$, this set can be splited into two sets, $\mathfrak{X}^{*}=\mathfrak{X}_{\text {int }}^{*} \cup \mathfrak{X}_{\text {ext }}^{*}$ where $\mathfrak{X}_{\text {int }}^{*} \cap \partial \Omega=\varnothing$ and $\mathfrak{X}_{\text {ext }}^{*} \subset \partial \Omega$. To each point $x_{\mathcal{K}^{*}} \in \mathfrak{X}_{\text {int }}^{*}$ (resp. $x_{\mathcal{K}^{*}} \in \mathfrak{X}_{\text {ext }}^{*}$ ), we associate the polygon $\mathcal{K}^{*} \in \mathfrak{M}^{*}$ (resp. $\left.\mathcal{K}^{*} \in \partial \mathfrak{M}^{*}\right)$ whose vertices are $\left\{x_{\mathcal{K}} \in \mathfrak{X}\right.$, such that $\left.x_{\mathcal{K}^{*}} \in \overline{\mathcal{K}}, \mathcal{K} \in \mathfrak{M}\right\}$ (resp. $\left\{x_{\mathcal{K}}\right\} \cup\left\{x_{\mathcal{K}} \in \mathfrak{X}\right.$, such that $\left.x_{\mathcal{K}^{*}} \in \overline{\mathcal{K}}, \mathcal{K} \in(\mathfrak{M} \cup \partial \mathfrak{M})\right\}$ ).

For all primal control volumes $\mathcal{K}$ and $\mathcal{L}$, we assume that $\partial \mathcal{K} \cap \partial \mathcal{L}$ is either empty or a common vertex or an edge of the primal mesh denoted by $\sigma=\mathcal{K} \mid \mathcal{L}$. We note by $\mathcal{E}$ the set of such edges, we also note $\sigma^{*}=\mathcal{K}^{*} \mid \mathcal{L}^{*}$ and $\mathcal{E}^{*}$ for the corresponding dual notations. 


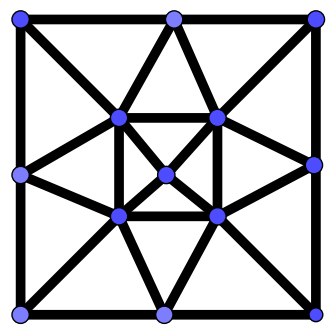

Primal cells $\mathcal{K} \in \mathfrak{M} \cup \partial \mathfrak{M}$

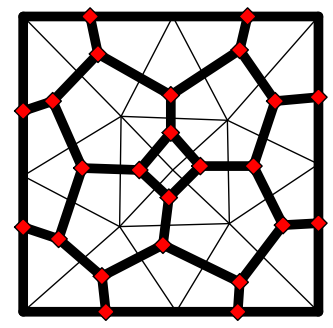

Dual cells $\mathcal{K}^{*} \in \mathfrak{M}^{\star} \cup \partial \mathfrak{M}^{\star *}$

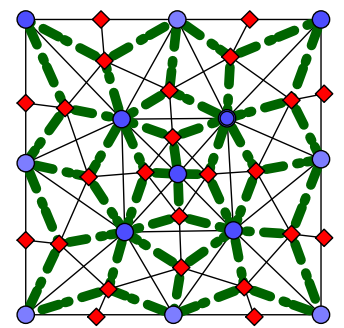

Diamond cell $\mathcal{D}_{\sigma, \sigma^{*}} \in \mathfrak{D}$

FiguRE 2. primal, dual and diamond meshes

Once the primal and dual control volumes are well defined, we give the diamonds cells $\mathcal{D}_{\sigma, \sigma^{*}}$ as the quadrangles whose diagonals are a primal edge $\sigma=\mathcal{K} \mid \mathcal{L}=\left(x_{\mathcal{K}^{*},} x_{\mathcal{L}^{*}}\right)$ and a dual edge $\sigma^{*}=\mathcal{K}^{*} \mid \mathcal{L}^{*}=\left(x_{\mathcal{K}}, x_{\mathcal{L}}\right)$, (see Figure 3). The quadrangle $\mathcal{D}_{\sigma, \sigma^{*}}$ degenerates into triangle. The set of diamond cells is denoted by $\mathfrak{D}$ and we have $\bar{\Omega}=\cup_{\mathcal{D} \in \mathfrak{D}} \overline{\mathcal{D}}$.

Notations: For any primal control volume $\mathcal{K} \in \mathfrak{M} \cup \partial \mathfrak{M}$, we note:

- $m_{\mathcal{K}}$ its Lebesgue measure,

- $\mathcal{E}_{\mathcal{K}}$ the set of its edges (if $\mathcal{K} \in \mathfrak{M}$ ), or the one-element set $\{\mathcal{K}\}$ if $\mathcal{K} \in \partial \mathfrak{M}$.

- $B_{\mathcal{K}}:=B\left(x_{\mathcal{K}}, \rho_{\mathcal{K}}\right) \cap \partial \Omega \subset \mathcal{K}$ for $\mathcal{K} \in \partial \mathfrak{M}, m_{B_{\mathcal{K}}}$, its length, the value of $\rho_{\mathcal{K}}$ is chosen so that the inclusion is verified.

- $B_{\mathcal{K}^{*}}:=B\left(x_{\mathcal{K}^{*}}, \rho_{\mathcal{K}^{*}}\right) \cap \partial \Omega \subset \mathcal{K}^{*}$ for $\mathcal{K}^{*} \in \partial \mathfrak{M}^{\star}, m_{B_{\mathcal{K}^{*}}}$ its length, the value of $\rho_{\mathcal{K}^{*}}$ is chosen so that the inclusion is verified.

- $\mathfrak{D}_{\mathcal{K}}=\left\{\mathcal{D}_{\sigma, \sigma^{*}} \in \mathfrak{D}, \sigma \in \mathcal{E}_{\mathcal{K}}\right\}$,

- $h_{\mathcal{K}}$ its diameter.

We will respectively note $: m_{\mathcal{K}^{*}}, \mathcal{E}_{\mathcal{K}^{*}}, \mathcal{D}_{\mathcal{K}^{*}}$ and $h_{\mathcal{K}^{*}}$.

We define $m_{\sigma}, m_{\sigma^{*}}, \vec{n}_{\sigma, \mathcal{K}}, \vec{n}_{\sigma^{*}, \mathcal{K}^{*}}, h_{\mathcal{D}}$ and $m_{\mathcal{D}}$ as follows:

- $m_{\sigma}$ the length of the primal edge $\sigma$,

- $m_{\sigma^{*}}$ the length of the dual edge $\sigma^{*}$,

- $\vec{n}_{\sigma \mathcal{K}}$ the unit vector normal to $\sigma$ oriented from $x_{\mathcal{K}}$ to $x_{\mathcal{L}}$,

- $\vec{n}_{\sigma^{*} \mathcal{K}^{*}}$ the unit vector normal to $\sigma^{*}$ oriented from $x_{\mathcal{K}^{*}}$ to $x_{\mathcal{L}^{*}}$,

- $h_{\mathcal{D}}$ its diameter,

- $m_{\mathcal{D}}$ its measure.

To complete our mathematical background of DDFV we propose the set of boundary diamond cell $\mathfrak{D}_{\text {ext }}$ as the set of diamond cells which possess one side included in $\partial \Omega$; the set of interior diamond cells is thus $\mathfrak{D}_{\text {int }}=\mathfrak{D} \backslash \mathfrak{D}_{\text {ext }}$. 


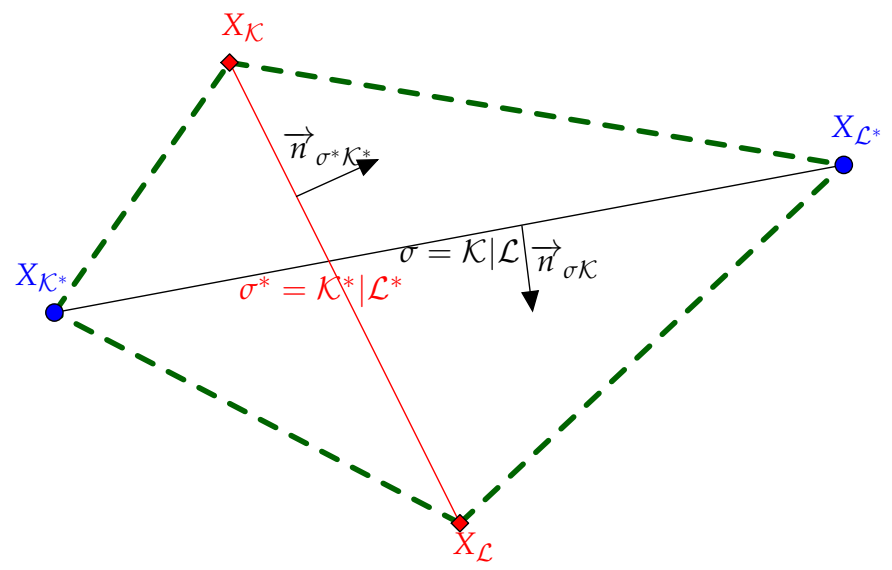

FiguRE 3. Diamond cell $\mathcal{D}_{\sigma, \sigma^{*}}$

2.2. Discrete unknowns and discrete mean-value projection. The DDFV method for the coupled Stokes-Darcy problem requires staggered unknowns. In $\Omega_{1}$ we associate to any primal cell $\mathcal{K} \in \mathfrak{M}_{1}$ an unknown value $u_{\mathcal{K}} \in \mathbb{R}^{2}$ and to any dual cell $\mathcal{K}^{*} \in \mathfrak{M}_{1}^{\star} \cup \partial \mathfrak{M}_{1}^{\star}$ an unknown value $u_{\mathcal{K}^{*}} \in \mathbb{R}^{2}$ for the velocity, and to any diamond $\mathcal{D} \in \mathfrak{D}_{1}$ an unknown value $p^{\mathcal{D}} \in \mathbb{R}$ for the pressure. In $\Omega_{2}$ we associate to any primal cell $\mathcal{K} \in \mathfrak{M}_{2}$ an unknown value $p_{\mathcal{K}} \in \mathbb{R}$ and to any dual cell $\mathcal{K}^{*} \in \mathfrak{M}_{2}^{\star} \cup \partial \mathfrak{M}_{2}^{\star}$ an unknown $p_{\mathcal{K}^{*}} \in \mathbb{R}$ for the pressure, and to any diamond cell $\mathcal{D} \in \mathfrak{D}_{2}$ an unknown value $u^{\mathfrak{D}} \in \mathbb{R}^{2}$ for the velocity. These unknowns are collected in families.

$$
\begin{gathered}
u^{\mathfrak{T}_{1}}=\left(\left(u_{\mathcal{K}}\right)_{\mathcal{K} \in \mathfrak{M}_{1}},\left(u_{\mathcal{K}^{*}}\right)_{\mathcal{K}^{*} \in\left(\mathfrak{M}_{1}^{\star} \cup \partial \mathfrak{M}_{1}^{\star}\right)}\right) \in\left(\mathbb{R}^{2}\right)^{\mathfrak{T}_{1}} \\
p^{\mathcal{D}_{1}}=\left(\left(p^{\mathcal{D}}\right)_{\mathcal{D} \in \mathfrak{D}_{1}}\right) \in(\mathbb{R})^{\mathfrak{D}_{1}} \\
p^{\mathfrak{T}_{2}}=\left(\left(p_{\mathcal{K}}\right)_{\mathcal{K} \in \mathfrak{M}_{2}}\left(p_{\mathcal{K}^{*}}\right)_{\mathcal{K}^{*} \in\left(\mathfrak{M}_{2}^{\star} \cup \partial \mathfrak{M}_{2}^{\star}\right)}\right) \in(\mathbb{R})^{\mathfrak{T}_{2}} \\
u^{\mathcal{D}_{2}}=\left(\left(u^{\mathcal{D}}\right)_{\mathcal{D} \in \mathfrak{D}_{2}}\right) \in\left(\mathbb{R}^{2}\right)^{\mathfrak{D}_{2}}
\end{gathered}
$$

We define now the mean-value projection for any vector field $v \in\left(H^{1}(\Omega)\right)^{2}$,

$$
\begin{aligned}
& P_{m}^{\partial \Omega} v=\left(\left(\frac{1}{m_{B_{\mathcal{K}}}} \int_{B_{\mathcal{K}}} v(x) d x\right)_{\mathcal{K} \in \partial \mathfrak{M}},\left(\frac{1}{m_{B_{k^{*}}}} \int_{B_{k^{*}}} v(x) d x\right)_{\mathcal{K}^{*} \in \partial \mathfrak{M}^{\star}}\right) \\
& P_{m}^{\mathfrak{M} v}=\left(\frac{1}{m_{k}} \int_{\mathcal{K}} v(x) d x\right)_{\mathcal{K} \in \mathfrak{M}^{\prime}}, P_{m}^{\mathfrak{M}^{*}} v=\left(\frac{1}{m_{k^{*}}} \int_{\mathcal{K}^{*}} v(x) d x\right)_{\mathcal{K}^{*} \in \mathfrak{M}^{\star}} .
\end{aligned}
$$

We finally gather these projections in the following notation

$$
P_{m}^{\mathcal{T}} v=\left(P_{m}^{\mathfrak{M}} v, P_{m}^{\mathfrak{M}^{\star}} v, P_{m}^{\partial \Omega} v\right), \quad \forall v \in\left(H^{1}(\Omega)\right)^{2} .
$$


2.3. Discrete operators. In this subsection, we define the discrete operators, gradient and divergence, which are needed in order to define and analyse the scheme, see [20] [15]

Definition 2.1. We define the discrete gradient operator $\nabla^{\mathfrak{D}_{1}}$ mapping vector field of $\left(\mathbb{R}^{2}\right)^{\mathcal{T}_{1}}$ into matrix fields of $\left(\mathcal{M}_{2}(\mathbb{R})\right)^{\mathfrak{D}_{1}}$ as follows:

$$
\begin{gathered}
\nabla^{\mathcal{D}_{1}} u^{\mathcal{T}_{1}}=\frac{1}{\sin \left(\alpha_{\mathcal{D}}\right)}\left(\frac{u_{\mathcal{L}}-u_{\mathcal{K}}}{m_{\sigma^{*}}} \otimes \vec{n}_{\sigma \mathcal{K}}+\frac{u_{\mathcal{L}^{*}}-u_{\mathcal{K}^{*}}}{m_{\sigma^{*}}} \otimes \vec{n}_{\sigma^{*} \mathcal{K}^{*}}\right), \quad \forall \mathcal{D} \in \mathfrak{D}_{1} . \\
\nabla^{\mathcal{D}_{1}} u^{\mathcal{T}_{1}}=\frac{1}{2 m_{\mathfrak{D}}}\left(m_{\sigma}\left(u_{\mathcal{L}}-u_{\mathcal{K}}\right) \otimes \vec{n}_{\sigma \mathcal{K}}+m_{\sigma^{*}}\left(u_{\mathcal{L}^{*}}-u_{\mathcal{K}^{*}}\right) \otimes \vec{n}_{\sigma^{*} \mathcal{K}^{*}}\right), \quad \forall \mathcal{D} \in \mathfrak{D}_{1} .
\end{gathered}
$$

And we define a consistent approximation of gradient operator denoted by $\nabla^{\mathfrak{D}_{2}}: p^{\mathcal{T}_{2}} \in \mathbb{R}^{\mathcal{T}_{2}} \longrightarrow\left(\nabla^{\mathcal{D}_{2}} p^{\mathcal{T}_{2}}\right)_{\mathcal{D} \in \mathfrak{D}_{2}} \in$ $\left(\mathbb{R}^{2}\right)^{\mathfrak{D}_{2}}$, as follows:

$$
\nabla^{\mathcal{D}_{2}} p^{\mathcal{T}_{2}}=\frac{1}{2 m_{\mathcal{D}}}\left(m_{\sigma}\left(p_{\mathcal{L}}-p_{\mathcal{K}}\right) \vec{n}_{\sigma \mathcal{K}}+m_{\sigma^{*}}\left(p_{\mathcal{L}^{*}}-p_{\mathcal{K}^{*}}\right) \vec{n}_{\sigma^{*} \mathcal{K}^{*}}\right), \quad \forall \mathcal{D} \in \mathfrak{D}_{2}
$$

Definition 2.2. We define the discrete gradient operator $\nabla^{\mathcal{T}}$ mapping a scalar fields $\mathbb{R}^{\mathfrak{D}_{1}}$ into vector fields in $\left(\mathbb{R}^{2}\right)^{\mathcal{T}_{1}}$ as follows

$$
\nabla^{\mathcal{T}} p^{\mathfrak{D}}=\operatorname{div}^{\mathcal{T}}\left(p^{\mathfrak{D}} I d\right), \quad \forall p^{\mathfrak{D}} \in \mathbb{R}^{\mathfrak{D}_{1}}
$$

Definition 2.3. We define the discrete divergence operator div ${ }^{\mathcal{T}}$ mapping vector field of $\left(\mathbb{R}^{2}\right)^{\mathfrak{D}_{1}}\left(\right.$ resp. $\left.\left(\mathbb{R}^{2}\right)^{\mathfrak{D}_{2}}\right)$ into scalar fields in $\mathbb{R}^{\mathcal{T}_{1}}$ (resp. $\mathbb{R}^{\mathcal{T}_{2}}$ ), as follows

$$
\begin{aligned}
& \operatorname{div} \mathcal{K}^{\mathcal{\zeta}^{\mathcal{D}}}=\frac{1}{m_{\mathcal{K}}} \sum_{\mathcal{D}_{\sigma, \sigma^{*}} \in \mathcal{D}_{\mathcal{K}}} m_{\sigma} \xi^{\mathfrak{D}} \vec{n}_{\sigma \mathcal{K}}, \forall \mathcal{K} \in \mathfrak{M}, \\
& \operatorname{div}^{\mathcal{K}^{*}} \xi^{\mathcal{D}}=\frac{1}{m_{\mathcal{K}^{*}}} \sum_{\mathcal{D}_{\sigma, \sigma^{*}} \in \mathcal{D}_{\mathcal{K}^{*}}} m_{\sigma^{*}} \mathfrak{\xi}^{\mathfrak{D}} \vec{n}_{\sigma^{*} \mathcal{K}^{*}}, \forall \mathcal{K}^{*} \in \mathfrak{M}^{\star},
\end{aligned}
$$

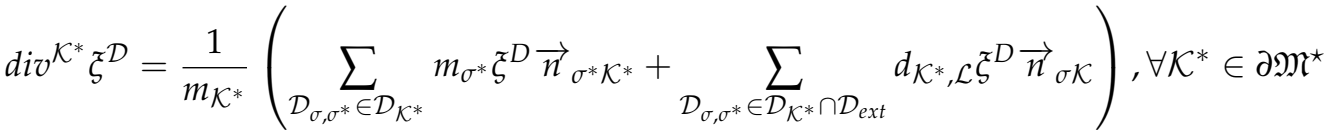

We mention here that there is no need to define the discrete divergence in both meshes $\mathcal{T}_{1}$ and $\mathcal{T}_{2}$ since there are similar, but as in [18] we give an other definition of the discrete divergence that we are going to need in Stokes equations:

The discrete divergence of a vector field of $\left(\mathbb{R}^{2}\right)^{\mathcal{T}_{1}}:$ div $^{\mathfrak{D}_{1}}: u^{\mathcal{T}_{1}} \in\left(\mathbb{R}^{2}\right)^{\mathcal{T}_{1}} \rightarrow\left(\operatorname{div}^{\mathfrak{D}_{1}} u^{\mathcal{T}}\right)_{D \in \mathfrak{D}} \in \mathbb{R}^{\mathfrak{D}}$ is defined as follows:

$$
\operatorname{div}^{\mathfrak{D}_{1}} u^{\mathcal{T}_{1}}=\operatorname{Tr}\left(\nabla^{\mathfrak{D}_{1}} u^{\mathcal{T}_{1}}\right)=\frac{1}{2 m_{\mathfrak{D}}}\left(m_{\sigma}\left(u_{\mathcal{L}}-u_{\mathcal{K}}\right) \cdot \vec{n}_{\sigma \mathcal{K}}+m_{\sigma^{*}}\left(u_{\mathcal{L}^{*}}-u_{\mathcal{K}^{*}}\right) \cdot \vec{n}_{\sigma^{*} \mathcal{K}^{*}}\right), \quad \forall \mathcal{D} \in \mathfrak{D}_{1}
$$

We give a DDFV mesh for the whole domain $\Omega=\Omega_{1} \cup \Omega_{2}$, with mention of the interface $\Gamma_{0}$ of contact between the two regions:

We present the subdomains $\Omega_{1}$ and $\Omega_{2}$ with two independent DDFV meshes $\mathcal{T}_{1}$ and $\mathcal{T}_{2}$. For each subdomain $\Omega_{i}$ we consider a DDFV mesh $\mathcal{T}_{i}=\left(\mathfrak{M}_{i}, \mathfrak{M}_{i}^{\star} \cup \partial \mathfrak{M}_{i}^{\star}\right)$ for $i=1,2$ and we associated diamond mesh $\mathfrak{D}_{i}$. For the boundary we use the notation $\partial_{0} \mathfrak{M}_{i}=\Gamma_{0}$ and $\left(\partial \mathfrak{M}_{i}^{\star}=\Gamma_{i}\right)_{i=1,2}$ so that :

$$
\partial \Omega_{1}=\Gamma_{0} \cup \partial \mathfrak{M}_{1}^{\star} \text {, and } \partial \Omega_{2}=\Gamma_{0} \cup \partial \mathfrak{M}_{2}^{\star} .
$$

In Darcy region we consider a well, proportionally with big dimensions just for visual cause:

We say that the two meshes are compatible in the following sense.

Definition 2.4. ([3]) The two meshes $\mathcal{T}_{1}$ and $\mathcal{T}_{2}$ are compatible if they verify the following two conditions: 


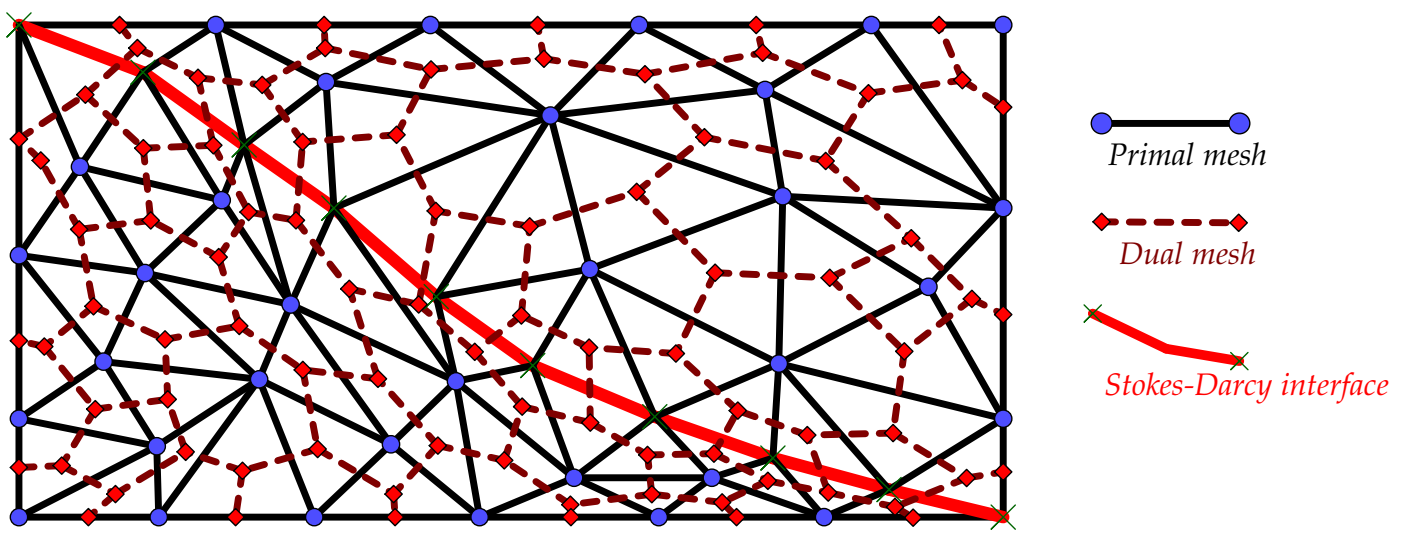

FiguRE 4. A DDFV mesh $\mathcal{T}$ of the whole domain $\Omega$
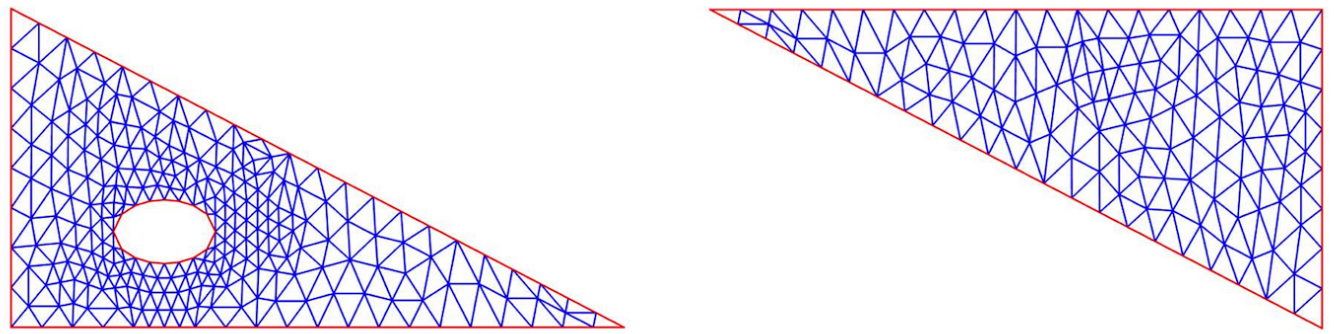

FiguRE 5. Two independent meshes $\mathcal{T}_{1}$ and $\mathcal{T}_{2}$ for both subdomains

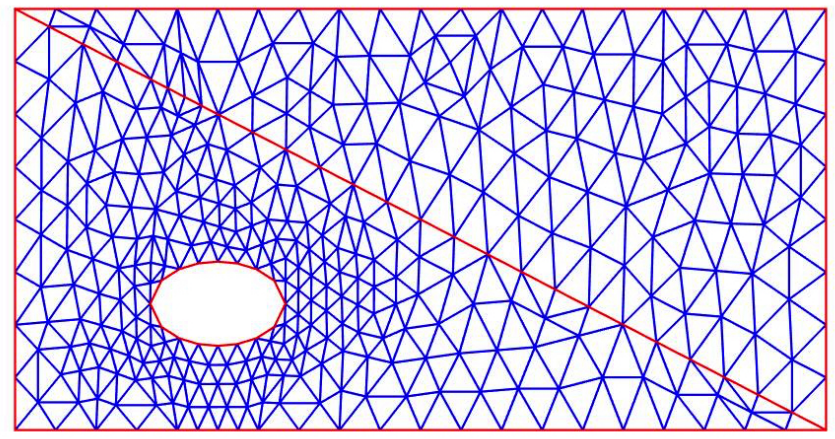

FIGURE 6. mesh for the whole domain $\Omega$

(1) The two meshes have the same vertices on $\Gamma_{0}$. This implies in particular that the two meshes have the same degenerate control volumes on $\Gamma_{0}$ i.e. $\partial_{0} \mathfrak{M}_{1}=\partial_{0} \mathfrak{M}_{2}, \partial_{0} \mathfrak{M}_{1}^{\star}=\partial_{0} \mathfrak{M}_{2}^{\star}$ and $\partial_{0} \mathfrak{D}_{1}=\partial_{0} \mathfrak{D}_{2}$ 
(2) The center $x_{\sigma}$ of a degenerate interface control volume $\sigma=\left[x_{K^{*}}, x_{L^{*}}\right] \in \partial_{0} \mathfrak{M}_{1}=\partial_{0} \mathfrak{M}_{2}$ is the intersection of $\left(x_{K^{*}}, x_{L^{*}}\right)$ and $\left(x_{K_{1}}, x_{K_{2}}\right)$, where $\mathcal{K}_{1} \in \mathfrak{M}_{1}$ and $\mathcal{K}_{2} \in \mathfrak{M}_{2}$ are the two primal control volumes such that $\sigma \subset \partial \mathcal{K}_{1}$ and $\sigma \subset \partial \mathcal{K}_{2}$.

Remark 2.1. We are given a DDFV mesh $\mathcal{T}$ of the whole domain $\Omega$ (see Figure 5) such that any primal control volume $\mathcal{K}$ is such that either $\mathcal{K} \subset \Omega_{1}$ or $\mathcal{K} \subset \Omega_{2}$. In that case, the construction of the two compatible meshes $\mathcal{T}_{i}$ only amounts to split into pieces the dual control volumes crossing the interface $\Gamma_{0}$ (see Figure 6).

\section{Main results}

3.1. Discretization of coupled Stokes-Darcy problem. We propose to resolve the coupled Stokes-Darcy problem using the finite volumes method. We give the standart problem:

As claimed in framework, we approximate the velocity on both vertices and centers of primal control volumes and the pressure on the diamond cells. We integrate the momentum conservation law of problem (1) on the interior primal mesh $\mathfrak{M}_{1}$ and on the dual mesh $\mathfrak{M}_{1}^{\star}$. The mass conservation equation is directly approached on the diamond mesh using the discrete operator div ${ }^{\mathfrak{D}}$. We impose the Dirichlet boundary conditions on $\mathfrak{M M}_{1}^{\star}$. Assuming for simplicity that $\mu=1$, the scheme for the problem (1) reads as follows:

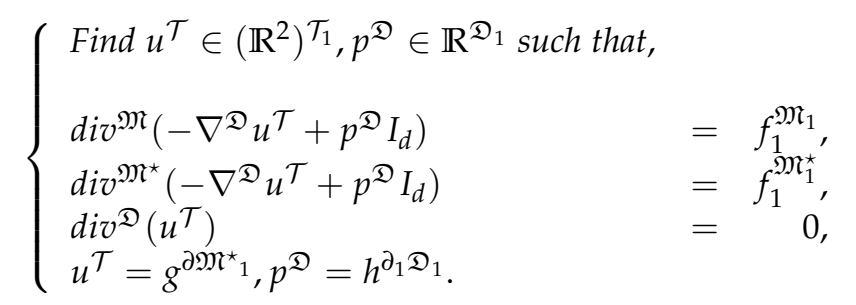

With $f_{1}^{\mathfrak{M}_{1}}=\mathbb{P}_{m}^{\mathfrak{M}_{1}} f$ and $f_{1}^{\mathfrak{M}_{1}^{\star}}=\mathbb{P}_{m}^{\mathfrak{M}_{1}^{\star}} f$.

Combining Darcy's law with the conservation of mass equation in (2) and assuming that $\mu=1$, we get the following equation:

$$
\left\{\begin{array}{cccc}
u_{2} & = & -K \nabla p_{2} \quad \text { in } \Omega_{2} \\
-\operatorname{div}\left(K \nabla p_{2}\right) & = & f_{2} & \text { in } \Omega_{2} \\
u_{2} & = & g_{2} & \text { on } \Gamma_{2},
\end{array}\right.
$$

The scheme for the problem 2 reads as follows:

$$
\left\{\begin{array}{lll}
\text { Find } u^{\mathfrak{D}} \in\left(\mathbb{R}^{2}\right)^{\mathfrak{D}_{2}}, p^{\mathcal{T}} \in \mathbb{R}^{\mathcal{T}_{2}} \text { such that, } & & \\
u^{\mathfrak{D}_{2}} & = & -K \cdot \nabla^{\mathfrak{D}} p^{\mathcal{T}_{2}}, \\
-\operatorname{div}^{\mathfrak{M}}\left(K . \nabla^{\mathfrak{D}} p^{\mathcal{T}_{2}}\right) & = & f^{\mathfrak{M}_{2}}, \\
-\operatorname{div}^{\mathfrak{M}^{\star}}\left(K \cdot \nabla^{\mathfrak{D}} p^{\mathcal{T}_{2}}\right) & = & f^{\mathfrak{M}_{2}^{\star}}, \\
u^{\mathfrak{D}}=h^{\partial_{2} \mathfrak{D}_{2}}, p^{\mathcal{T}}=g^{\partial \mathfrak{M}_{2}^{\star}} & &
\end{array}\right.
$$

Note the absence of information on velocity and pressure on the interface $\Gamma_{0}$, and to overcome this problem, we have used the Schwarz method. The algorithm consists in constructing sequences $\left(u_{n}^{1}\right)_{n},\left(u_{n}^{2}\right)_{n} \subset\left(\mathbb{R}^{2}\right)^{\mathcal{T}_{1}} \times\left(\mathbb{R}^{2}\right)^{\mathfrak{D}_{2}}$ and $\left(p_{n}^{1}\right)_{n},\left(p_{n}^{2}\right)_{n} \subset \mathbb{R}^{\mathfrak{D}_{1}} \times \mathbb{R}^{\mathcal{T}_{2}}$

Choose Initializations $u_{0}^{1}, u_{0}^{2}, p_{0}^{1}$ and $p_{0}^{2}$

For all $n \geq 0$, Perform successively: 


$$
\begin{cases}\text { Find }\left(u_{n+1}^{1}\right)^{\mathcal{T}} \in\left(\mathbb{R}^{2}\right)^{\mathcal{T}_{1}} \text { and }\left(p_{n+1}^{1}\right)^{\mathfrak{D}} \in \mathbb{R}^{\mathfrak{D}_{1}} \text { such that, } & \\ \operatorname{div}^{\mathfrak{M}}\left(-\nabla^{\mathfrak{D}}\left(u_{n+1}^{1}\right)^{\mathcal{T}_{1}}+\left(p_{n+1}^{1}\right)^{\mathfrak{D}_{1}} I d\right) & =f_{1}^{\mathfrak{M}_{1}}, \\ \operatorname{div}^{\mathfrak{M}^{\star}}\left(-\nabla^{\mathfrak{D}}\left(u_{n+1}^{1}\right)^{\mathcal{T}_{1}}+\left(p_{n+1}^{1}\right)^{\mathfrak{D}_{1}} I d\right) & =f_{1}^{\mathfrak{M}_{1}^{\star}} \\ \operatorname{div}^{\mathfrak{D}}\left(\left(u_{n+1}^{1}\right)^{\mathcal{T}_{1}}\right) & =0, \\ u_{n+1}^{1}=g^{\partial \mathfrak{M}_{1}^{\star}}, p_{n+1}^{1}=h^{\partial_{1} \mathfrak{D}_{1}} \text { on } \Gamma_{1} & \\ \left(u_{n+1}^{1}\right)^{\partial_{0} \mathfrak{M}_{1}^{\star}}=\mathbb{P}^{\partial_{0} \mathfrak{M}_{1}^{\star}}\left(u_{n}^{2}\right)^{\partial_{0} \mathfrak{D}_{2}}, \quad\left(p_{n+1}^{1}\right)^{\partial_{0} \mathfrak{D}_{1}}=\mathbb{P}^{\partial_{0} \mathfrak{D}_{1}}\left(p_{n}^{2}\right)^{\partial_{0} \mathcal{T}_{2}} & \end{cases}
$$

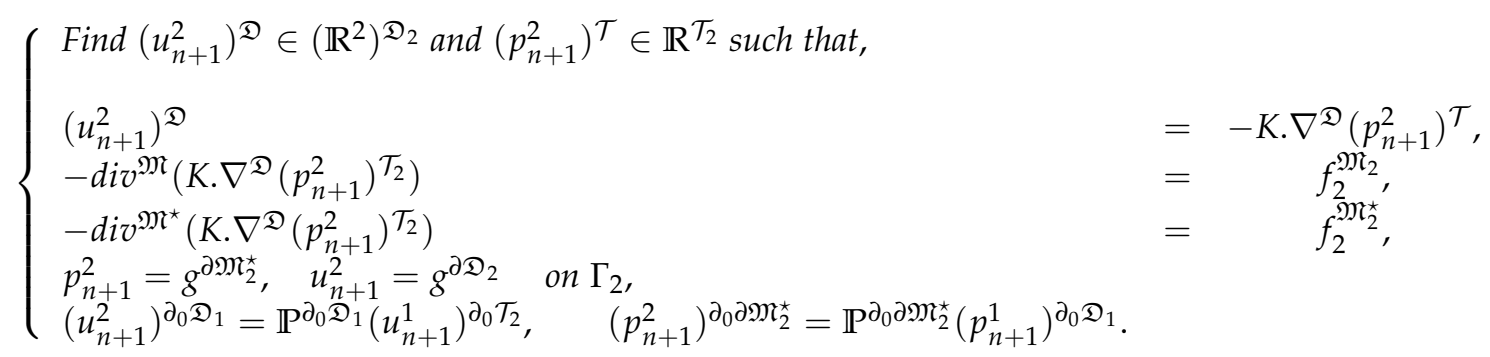

Stop at convergence when the velocity $u_{n}^{1}$ and $u_{n}^{2}$ (after interpolation) are sufficiently close (resp. the pressure) on $\Gamma_{0}$. Since the the approximation of pressure in $\Omega_{2}$ is over primal and dual control volume while in $\Omega_{2}$ we approximate pressure on diamonds, we need to introduce an interpolation operator on $\left(\partial_{0} \mathfrak{D}_{i}\right)_{i=1,2}$.

We propose te following operator on diamonds $\mathbb{P}^{\partial_{0} \mathfrak{D}}$, it's a mapping from $\mathbb{R}^{\mathcal{T}}$ to $\mathbb{R}^{\mathfrak{D}}$ defined for all $p^{\mathcal{T}} \in \mathbb{R}^{\mathcal{T}}$ by $\mathbb{P}^{\partial_{0} \mathfrak{D}_{i}}\left[p^{\mathcal{T}}\right]=\left(\mathbb{P}^{\partial_{0} \mathfrak{D}_{i}}\left(p^{\mathcal{T}}\right)\right)_{\mathcal{D} \in \mathfrak{D}}$ where for $\mathcal{D} \in \partial \mathfrak{D}$, whose vertices are $x_{\mathcal{K}}, x_{\mathcal{K}^{*}}$ and $x_{\mathcal{L}^{*}}$ see $[5]$ :

$$
\mathbb{P}^{\partial_{0} \mathfrak{D}_{i}}\left(p^{\mathcal{T}}\right)=\frac{1}{3}\left(p_{\mathcal{K}}+p_{\mathcal{K}^{*}}+p_{\mathcal{L}^{*}}\right)
$$

then we propose as a stop criterion the following error through diamonds:

$$
\left\|\left(p^{1}\right)^{\partial_{0} \mathfrak{D}_{1}}-\mathbb{P}^{\partial_{0} \mathfrak{D}_{2}}\left(p^{\mathcal{T}_{2}}\right)\right\|_{L^{\infty}}
$$

\subsection{Numerical examples.}

Examples. Example 1 Let $\Omega=\Omega_{1} \cup \Omega_{2}$ with $\Omega_{1}=\left\{(x, y) \in \mathbb{R}^{2}: 0 \leq x \leq 1\right.$ and $\left.0 \leq y \leq 1-x\right\}$ and $\Omega_{2}=\Omega^{*} \backslash C$, such that $\Omega^{*}=\left\{(x, y) \in \mathbb{R}^{2}: 0 \leq x \leq 1\right.$ and $\left.1-x \leq y \leq 1\right\}$ and $C=\left\{(x, y) \in \mathbb{R}^{2}:(x-0.2)^{2}+(y-0.2)^{2}=(0.1)^{2}\right\}$

For simplicity we take the kinematic viscosity of the fluid $\mu=1$ and so that we can verify our method we propose in a first example where the tensor of permeability is $K=I$. As a stop criterion for the convergence of Schwarz algorithm, we require the $L^{\infty}$-norm of the difference of pressure $\left\|\left(p^{1}\right)^{\partial_{0} \mathfrak{D}_{1}}-\mathbb{P}^{\partial_{0} \mathfrak{D}_{2}}\left(p^{2}\right)^{\mathcal{T}_{2}}\right\|$ to be smaller than $10^{-8}$ (resp. the velocity).In practice the number of iterations is never bigger than 10.

We propose the following problem in $\Omega_{1}$

$$
\left\{\begin{array}{cccc}
-\Delta u_{1}+\nabla p_{1} & = & f_{1} & \text { in } \Omega_{1} \\
\nabla \cdot u_{1} & = & 0 & \text { in } \Omega_{1} \\
u_{1}=u_{1}^{\text {ex }} & \text { and } & p_{1}= & p_{1}^{\text {ex }} \text { on } \Gamma_{1}
\end{array}\right.
$$

Such that

$$
f_{1}(x, y)=\left((2 x-1)(1-y) \quad ; \quad x(1-x)+(y-1)^{2}-4\right)
$$


In $\Omega_{2}$ we consider Darcy problem:

$$
\left\{\begin{array}{ccc}
u_{2}+K \nabla p_{2} & = & 0 \text { in } \Omega_{2} \\
\nabla \cdot u_{2} & = & f_{2} \text { in } \Omega_{2} \\
u_{2}=u_{2}^{e x} & \text { and } & p_{2}=p_{2}^{\text {ex }} \text { on } \Gamma_{2} \cup \partial C
\end{array}\right.
$$

Such that $f_{2}=0$

We present the exact solution of pressure and viscosity:

$$
\begin{gathered}
u_{1}^{e x}(x, y)=u_{2}^{e x}(x, y)=\left((2 x-1)(y-1) \quad ; \quad x(x-1)-(y-1)^{2}\right), \text { for }(x, y) \in \Omega_{1} \\
p_{1}^{e x}(x, y)=p_{2}^{e x}(x, y)=\left(x(1-x)(y-1)+\frac{(y-1)^{3}}{3}\right), \text { for }(x, y) \in \Omega,
\end{gathered}
$$

We give the exact solution and the numerical solution in $\Omega_{1}$ :

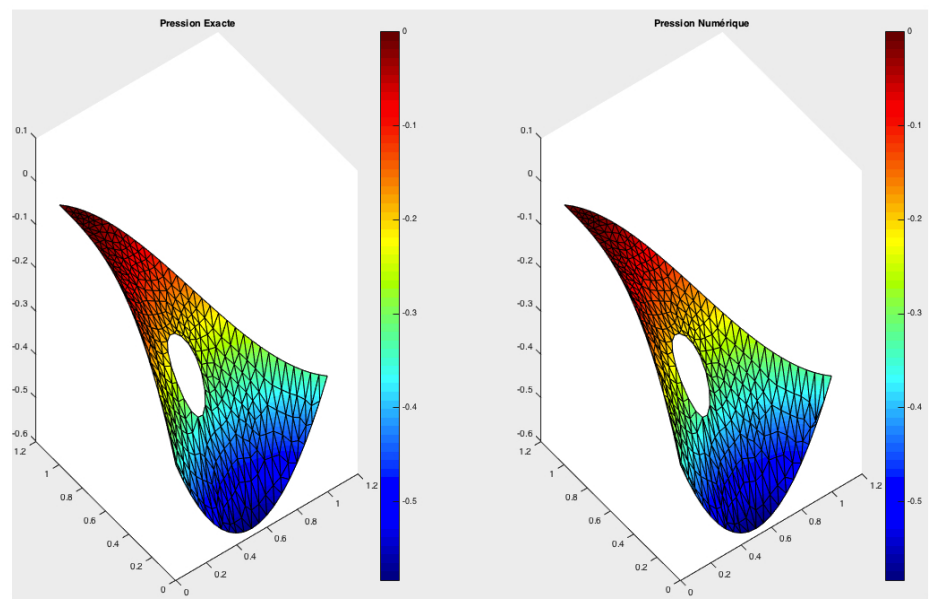

FIGURE 7. Exact solution and numerical solution of pressure in $\Omega_{1}$ 

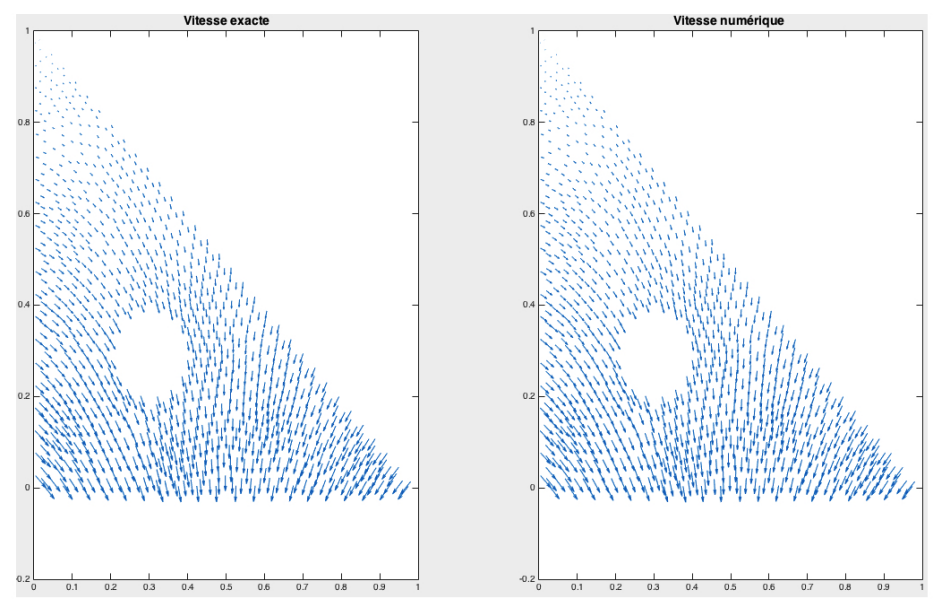

FIGURE 8. Exact solution and numerical solution of velocity in $\Omega_{1}$

And we give the exact solution and the numerical solution in $\Omega_{2}$ :
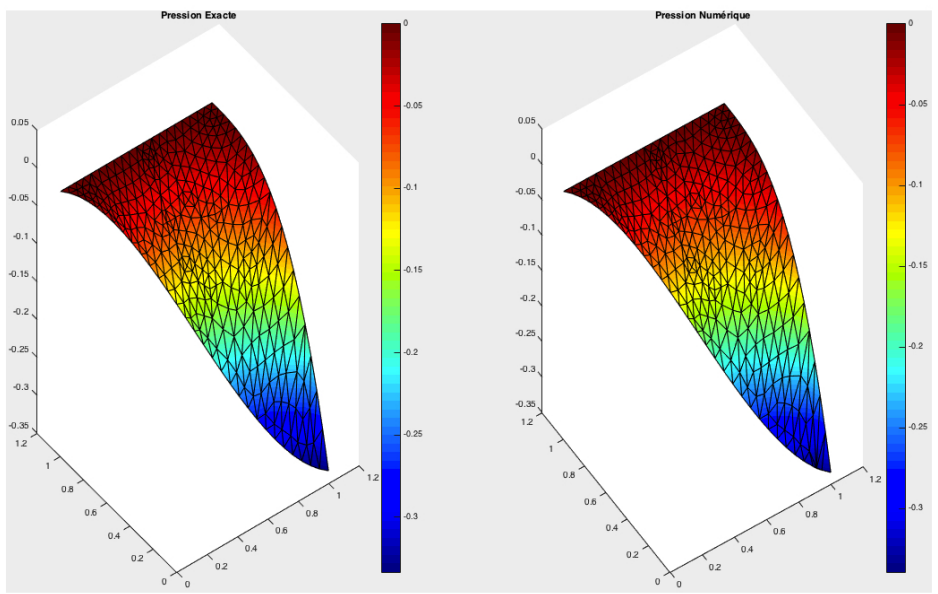

FIGURE 9. Exact solution and numerical solution of pressure in $\Omega_{2}$ 
A DISCRETE DUALITY FINITE VOLUME METHOD FOR COUPLING DARCY AND STOKES EQUATIONS
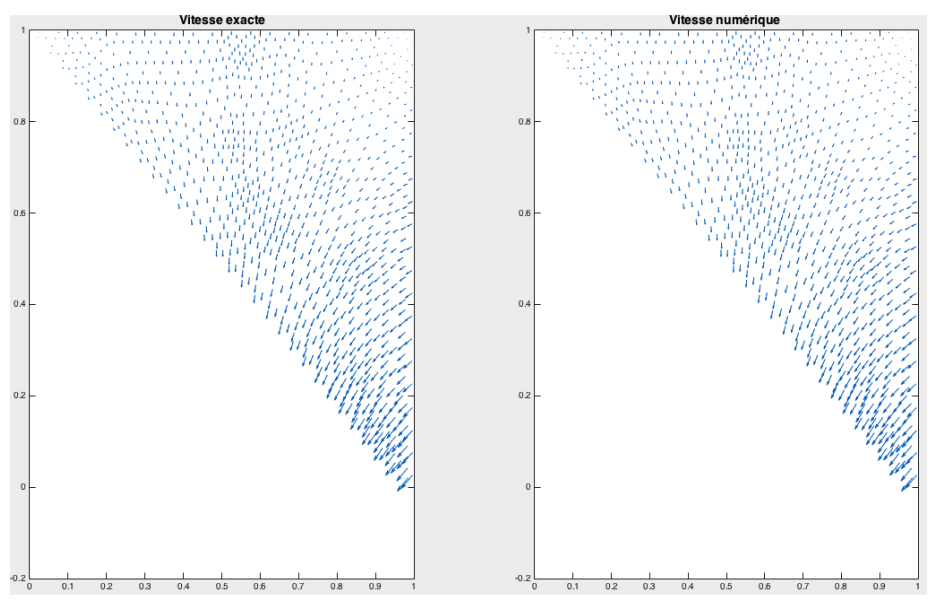

FIGURE 10. Exact solution and numerical solution of velocity in $\Omega_{2}$

We present the numerical approximation of pressure in $\Omega_{1}$ and $\Omega_{2}$ :
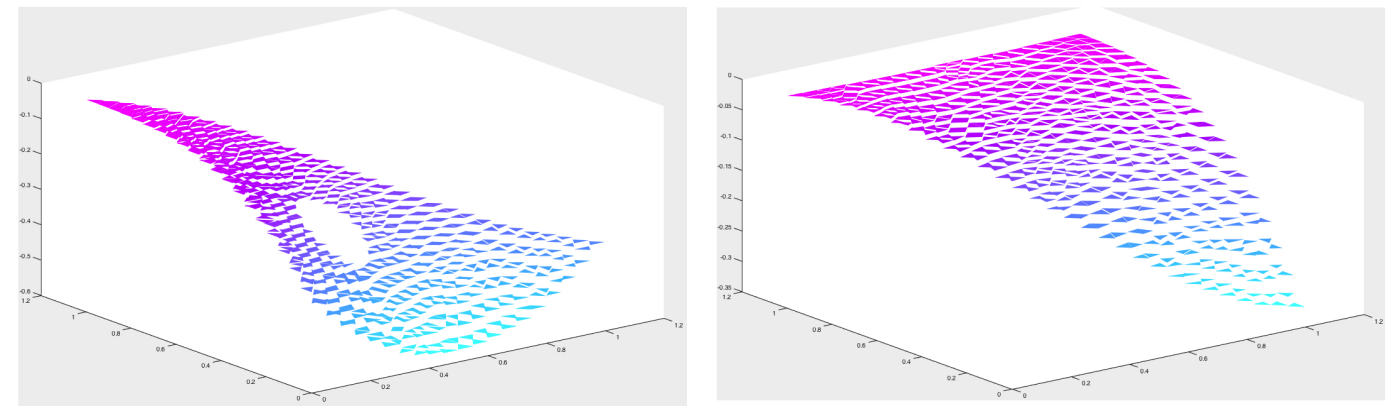

FIGURE 11. Stokes approximation of pressure and Darcy approximation of pressure

Then we present the numerical approximation of pressure in the whole domain $\Omega$ 


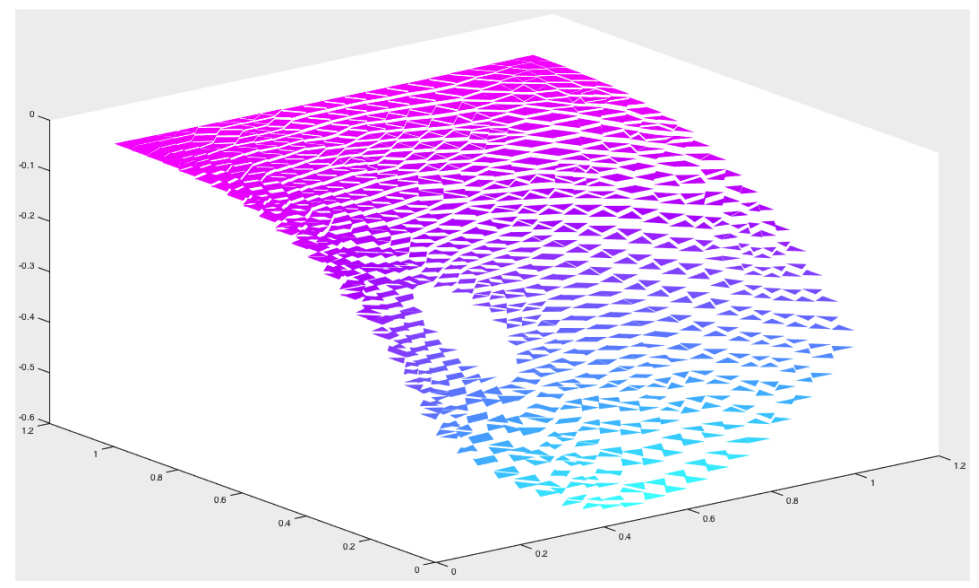

FIGURE 12. Stokes-Darcy approximation of pressure

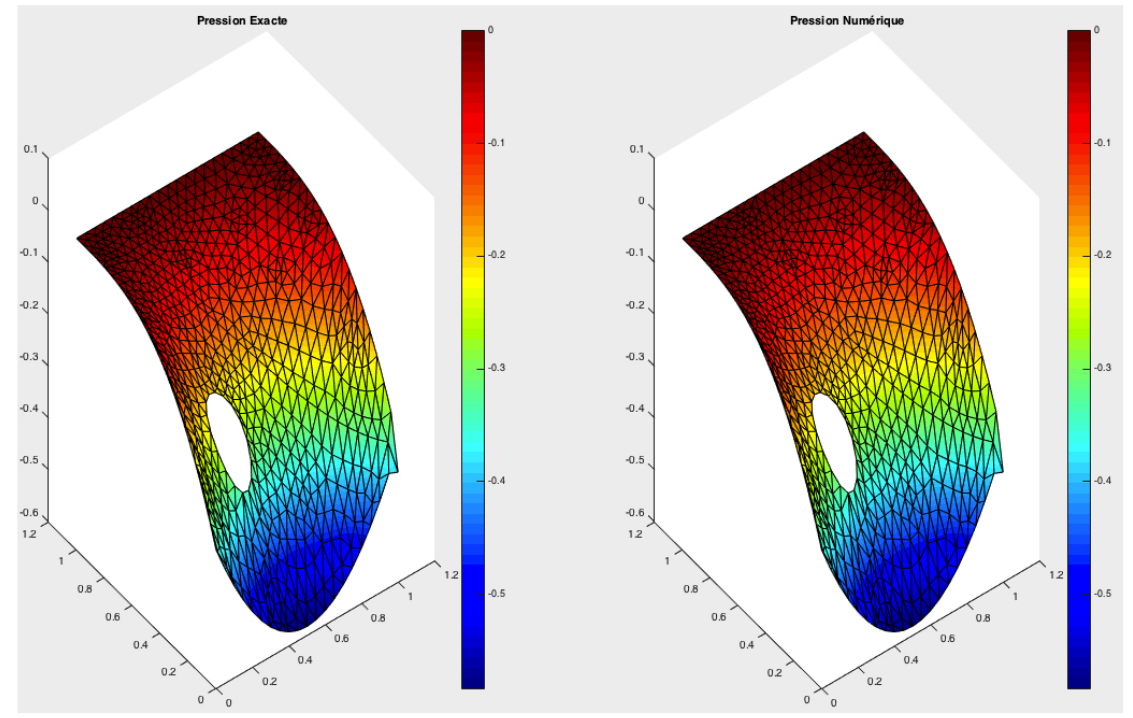

FIGURE 13. Exact solution and numerical solution of pressure in $\Omega$ 

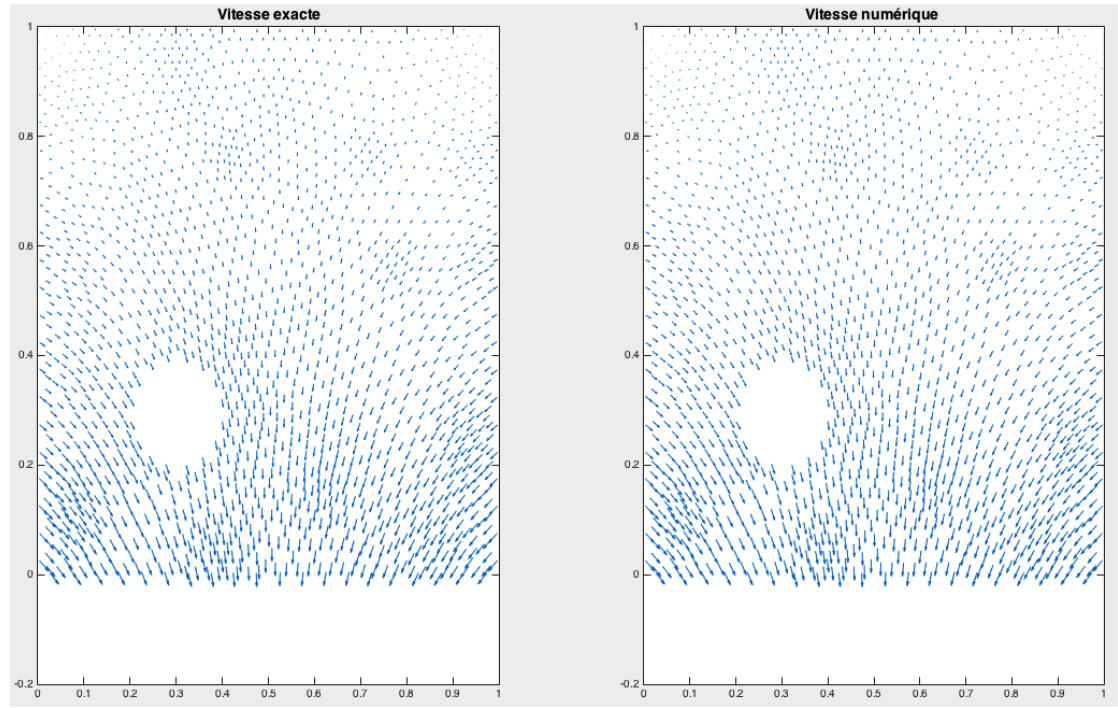

FIGURE 14. Exact solution and numerical solution of velocity in $\Omega$

The errors between the exact solution and the numerical solution for pressure and velocity:

\begin{tabular}{cccc||c||c}
\hline h mesh & primal cells & dual cells & diamond cells & $U_{e x}-U_{n u} \|_{\infty}$ & $\left\|U_{e x}-U_{n u}\right\|_{L^{2}}$ \\
\hline 0.4787 & 20 & 17 & 36 & 0.2011 & 0.1010 \\
0.1197 & 334 & 188 & 521 & 0.0657 & 0.0192 \\
0.0598 & 1336 & 709 & 2044 & 0.0332 & 0.0113 \\
0.0299 & 5344 & 2753 & 8096 & 0.0164 & 0.0062 \\
0.0150 & 20992 & 10657 & 31648 & 0.0081 & 0.0032 \\
\hline \hline h mesh & primal cells & dual cells & diamond cells & $P_{e x}-P_{n u} \|_{\infty}$ & $\left\|P_{e x}-P_{n u}\right\|_{L^{2}}$ \\
\hline 0.4787 & 20 & 17 & 36 & 0.1124 & 0.0836 \\
0.1197 & 334 & 188 & 521 & 0.0384 & 0.0234 \\
0.0598 & 1336 & 709 & 2044 & 0.0159 & $7.3420 E-03$ \\
0.0299 & 5344 & 2753 & 8096 & 0.0089 & $3.5811 E-03$ \\
0.0150 & 20992 & 10657 & 31648 & 0.0055 & $1.9802 E-03$ \\
\hline
\end{tabular}

Example 2

We keep the same domains $\Omega_{1}$ and $\Omega_{2}$ but this time we propose the tensor of permeability which is symmetric positive definite in $\Omega$ as:

$$
K=\left(\begin{array}{cc}
1+x & -x y \\
-x y & 1+y
\end{array}\right)
$$

In $\Omega_{1}$, we propose the following problem:

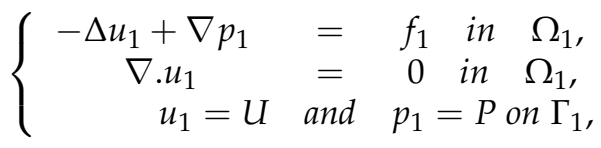


And we propose the Darcy problem in $\Omega_{2}$ :

$$
\left\{\begin{array}{cccc}
u_{2}+K \nabla p_{2} & = & 0 \text { in } \Omega_{2} \\
\nabla \cdot u_{2} & = & 0 \text { in } \Omega_{2} \\
u_{2}=U & \text { and } & p_{2}=P \text { on } \Gamma_{2} \cup \partial C
\end{array}\right.
$$

Such that:

$$
\begin{gathered}
f_{1}=\left(\frac{x^{2} y(x-1)}{(x+1)(y+1)-x^{2} y^{2}}, \frac{x^{3}-x+2}{(x+1)(y+1)-x^{2} y^{2}}\right), \\
P=x(x-1)(y-1)-\frac{(y-1)^{3}-2}{3} \text { and } U=(0, x(1-x))
\end{gathered}
$$

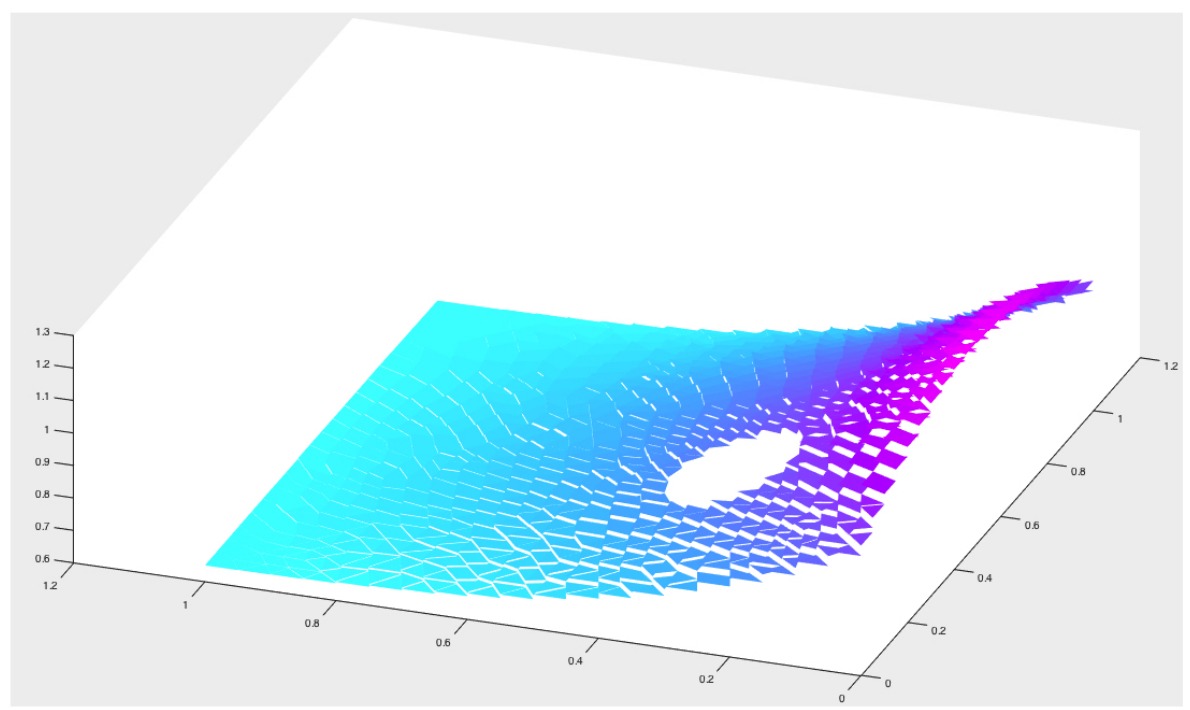

FIGURE 15. Numerical solution of pressure in $\Omega$ 


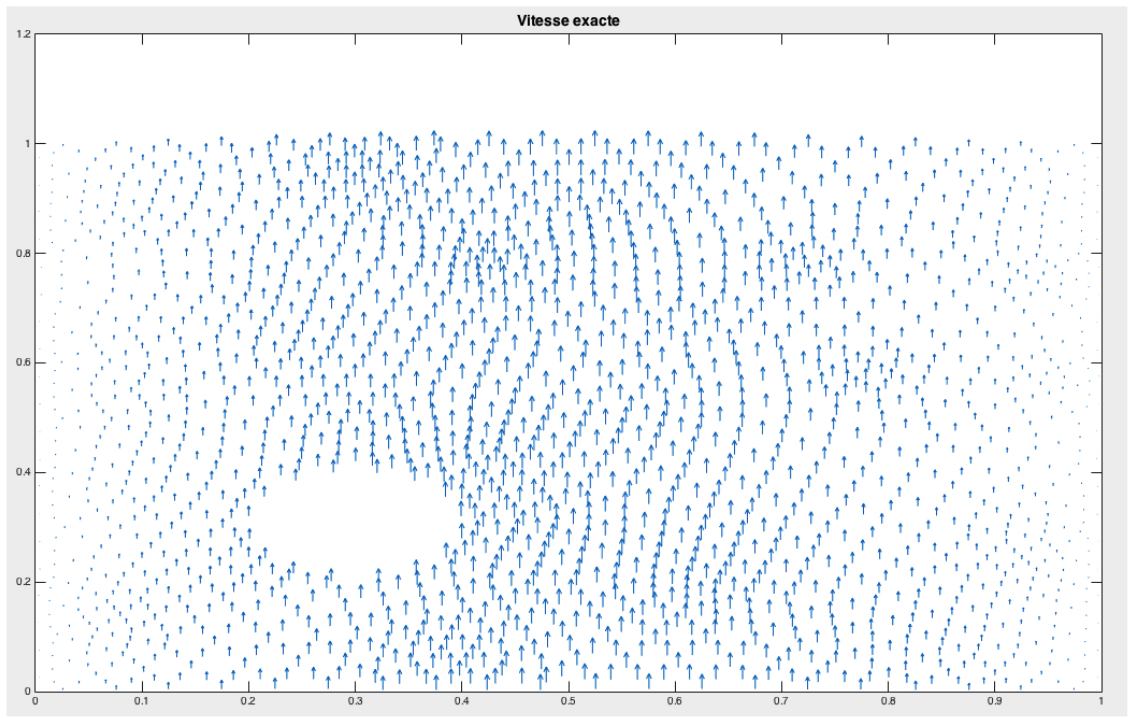

FIGURE 16. Numerical solution of velocity in $\Omega$

\section{Conclusions}

We presented the Discrete Duality Finite Volume method for the coupling of Stokes and Darcy flow, by the use of Schwarz method to threat the interface problem. We presented an error norm estimate which is optimal with respect to the approximation spaces. We hope proving the convergence of the scheme see [13] and finding a more strong model by including more conditions on the interface $\Gamma$, such as Beavers-Joseph-Saffman[12] which will be postponed to a future article.

\section{References}

[1] B. Andreianov, F. Boyer, and F. Hubert. Discrete duality finite volume schemes for leray- lions- type elliptic problems on general 2d meshes. 23(1):145-195.

[2] Y. Boubendir and S. Tlupova. Stokesdarcy boundary integral solutions using preconditioners. 228(23):86278641.

[3] F. Boyer, F. Hubert, and S. Krell. Non-overlapping schwarz algorithm for solving 2d m-DDFV schemes. 30(4):Pp 1062-1100.

[4] M. Cai, M. Mu, and J. Xu. Preconditioning techniques for a mixed stokes/darcy model in porous media applications. 233(2):346-355.

[5] C. Cancs, C. Chainais-Hillairet, and S. Krell. Numerical analysis of a nonlinear free-energy diminishing discrete duality finite volume scheme for convection diffusion equations. 18(3):407-432.

[6] P. Chidyagwai and B. Rivire. On the solution of the coupled navierstokes and darcy equations. 198(47):38063820.

[7] M. Correa and A. Loula. A unified mixed formulation naturally coupling stokes and darcy flows. 198(33):2710-2722.

[8] S. Delcourte, K. Domelevo, and P. Omnes. A discrete duality finite volume approach to hodge decomposition and divcurl problems on almost arbitrary twodimensional meshes. 45(3):1142-1174.

[9] M. Discacciati. Domain decomposition methods for the coupling of surface and groundwater flows.

[10] M. Discacciati, E. Miglio, and A. Quarteroni. Mathematical and numerical models for coupling surface and groundwater flows. 43(1):57-74. 
[11] K. Domelevo and P. Omnes. A finite volume method for the laplace equation on almost arbitrary twodimensional grids. 39(6):1203-1249.

[12] G. Du, Y. Hou, and L. Zuo. A modified local and parallel finite element method for the mixed stokesdarcy model. 435(2):1129-1145.

[13] M. Gander, L. Halpern, F. Hubert, and S. Krell. Optimized schwarz methods for anisotropic diffusion with discrete duality finite volume discretizations. page 34 .

[14] M. Hellou, J. Martinez, and M. El Yazidi. Stokes flow through microstructural model of fibrous media. 31(1):97-103.

[15] F. Hermeline. A finite volume method for the approximation of diffusion operators on distorted meshes. 160(2):481-499.

[16] A. R. A. Khaled and K. Vafai. The role of porous media in modeling flow and heat transfer in biological tissues. 46(26):4989-5003.

[17] J. M. V. A. Koelman and M. Nepveu. Darcy flow in porous media: Cellular automata simulations. In Numerical Methods for the Simulation of Multi-Phase and Complex Flow, number 398 in Lecture Notes in Physics, pages 136-145. Springer Berlin Heidelberg.

[18] S. Krell. Stabilized DDFV schemes for stokes problem with variable viscosity on general $2 \mathrm{~d}$ meshes. 27(6):1666-1706.

[19] W. J. Layton, F. Schieweck, and I. Yotov. Coupling fluid flow with porous media flow. 40(6):2195-2218.

[20] P. Omnes. Dveloppement et analyse de mthodes de volumes finis.

[21] H. Rui and R. Zhang. A unified stabilized mixed finite element method for coupling stokes and darcy flows. 198(33):2692-2699.

[22] J. Urquiza, D. N’Dri, A. Garon, and M. Delfour. Coupling stokes and darcy equations. 58(5):525-538. 